\title{
Abiraterone treatment improves overall survival in patients with mCRPC
}

New research published in the Lancet Oncology confirms that abiraterone, a hormonal therapy that targets the androgen receptor signalling pathway, significantly improves overall survival in patients with metastatic castration-resistant prostate cancer (mCRPC). "In previous interim analyses, strong trends in improved overall survival were observed, but results did not cross the prespecified efficacy boundary for statistical significance," explains Charles Ryan, lead author of the study.

In a double-blind, randomized trial, patients with chemotherapy-naive prostate cancer were assigned to receive abiraterone plus prednisone or placebo plus prednisone for a maximum treatment period of 60 months. After a median follow-up of 49.2 months, patients receiving abiraterone had significantly longer overall survival compared with those receiving placebo (34.7 months versus 30.3 months, $P=0.0033)$. Drug-related adverse events were generally more common in the abiraterone group, resulting in treatment discontinuation in $7 \%$ of patients compared with $4 \%$ of patients receiving placebo.

In conclusion, abiraterone treatment represents an improvement in our ability to treat patients with mCRPC. On future directions for research in this area, Ryan adds, "accounting for subsequent therapies, but also determining the relative efficacy of therapies based on when, in a sequenced approach or in combination, they might be administered, remains a major challenge."

Peter Sidaway

This article has also been published in Nature Reviews Clinical Oncology (doi:10.1038/nrclinonc.2015.14)

Original article Ryan, C. J. et al. Abiraterone acetate plus prednisone versus placebo plus prednisone in chemotherapynaive men with metastatic castration-resistant prostate cancer (COU-AA-302): final overall survival analysis of a randomised, double-blind, placebo-controlled phase 3 study. Lancet Oncol. doi:10.1016/S1470-2045(14)71205-7 